\title{
Pattern classification of Myo-Electrical signal during different Maximum Voluntary Contractions: A study using BSS techniques
}

\author{
Ganesh R. Naik, Dinesh K. Kumar, Sridhar P. Arjunan \\ Electrical and Computer Engineering, RMIT University, Melbourne, Australia, ganesh.naik@rmit.edu.au
}

\begin{abstract}
The presence of noise and cross-talk from closely located and simultaneously active muscles is exaggerated when the level of muscle contraction is very low. Due to this the current applications of surface electromyogram (sEMG) are infeasible and unreliable in pattern classification. This research reports a new technique of sEMG using Independent Component Analysis (ICA). The technique uses blind source separation (BSS) methods to classify the patterns of Myo-electrical signals during different Maximum Voluntary Contraction (MVCs) at different low level finger movements. The results of the experiments indicate that patterns using ICA of sEMG is a reliable $(p<0.001)$ measure of strength of muscle contraction even when muscle activity is only $20 \%$ MVC. The authors propose that ICA is a useful indicator of muscle properties and is a useful indicator of the level of muscle activity.
\end{abstract}

Keywords: Surface Electromyography, Independent component Analysis, Blind source separation, Gestures, Maximum Voluntary Contractions, RMS, Motor Unit Action Potential

\section{INTRODUCTION}

$\mathrm{S}$ URFACE ELECTROMYOGRAPHY (sEMG) is the electrical recording of the muscle activity from the surface. It is closely related to the strength of muscle contraction and is a useful to estimate the strength of contraction of the muscle. But the presence of artefacts and cross-talk, especially at low level of muscle activity make the recordings unreliable. Spectral and temporal overlap can make the removal of artefacts and noise, or separation of relevant signals from other bioelectric signals extremely difficult. Most commonly used feature of sEMG to measure the strength of contraction of a muscle is root mean square (RMS) of sEMG. RMS is a global parameter and is dependent on the number of motor unit action potential (MUAP), size of the motor units and the location of the muscle. Other similar features of sEMG commonly used include windowed integration and zero crossing count [1].

To exploit the relationship between the magnitude and/ or the spectral content of SEMG with force of contraction of the muscles, various analogous measures such as root mean square (RMS), integral of the signal, and wavelet coefficients have been used to classify the signal against the desired movement and/or posture [2]-[4]. RMS of sEMG has been considered for controlling and for virtual reality entertainment [5]-[8]. These features are easy to implement and are a good measure of the strength of muscle activity when there is a single active muscle that has high level of muscle activity. However these measures are not suitable when the muscle activity is very small and there are multiple muscles that are simultaneously active.

To identify low level forearm movements and actions that are a result of multiple active muscles, relative muscle activity from the different muscles in the forearm has to be identified. For this purpose, sEMG needs to be recorded using multiple electrodes. However due to the close proximity of the different active muscles, each of these electrodes record muscle activity from different muscles, referred to as cross talk. In case of the fore arm, this is always a problem, and this is further exaggerated when the muscle activity is weak like during maintained isometric gestures. Spectral and temporal overlap makes the use of conventional filtering quite useless. Another difficulty of such identification of movements at low level of contraction is the poor signal to noise ratio for sEMG recording when muscle activity is small.

To reliably identify the small movements and gesture of the hand, there is need to decompose sEMG into muscle activity originating from the different muscles. Spectral and temporal filtering is not suitable for this because of overlapping spectra and simultaneously active muscles. Blind source separation (BSS) techniques have recently been developed and these provide a solution for such a situation. BSS techniques such as Independent Component Analysis (ICA) have found numerous applications in audio and biosignal processing disciplines. ICA of sEMG has been proposed for identifying cortical activation related to armmovement combinations in 2000 [9]. Mckewon and Radtke demonstrated a method for phase and tonic coupling between EEG and EMG using ICA [10]. Decomposition of sEMG into motor unit action potentials (MUAP) originating from different muscles and motor units has been reported in 2004 [11]. The authors have demonstrated the separation of sEMG using ICA into individual muscle activity for the hand gesture identification [12, 13]; however separating the low level muscle activities using ICA is always a challenging task as numerous muscles active at the same time even during a small muscle contraction. This paper reports the research conducted to separate the low level muscle activities using ICA for different MVCs of EMG.

\section{MATERIALS AND METHODS}

\section{A. Blind Source Separation}

Blind source separation (BSS) are a set of techniques used for recovering signals from unobserved 'sources' from observed mixtures with no information available regarding the mixing matrix or the sources. Typically each sensor receives a mixture of signals from the various sources. The simplest BSS 
technique aims at transforming an input vector into a signal space in which the signals are statistically independent [14, 15]. This is achieved iteratively by reducing a cost function that is related to dependence within the different signals.

The simple BSS model assumes that the mixing process is linear, and is expressed as:

$$
x(t)=A s(t)
$$

where $\quad x(t)=\left[x_{1}(t), \ldots, x_{n}(t)\right]^{T}$ are the recordings, $s(t)=\left[s_{1}(t), \ldots, s_{n}(t)\right]$ the original signals, and $A$ is the $n \times n$ mixing matrix. This mixing matrix and each of the original signals are unknown. To separate the recordings to the original signals (estimated original signals $s$ ), the task is to estimate an un-mixing matrix $W$ so that:

$$
s(t)=W x(t)=W A s(t)
$$

ICA iteratively determines the un-mixing matrix $W$ and thus estimates the corresponding independent signals $s$ from the observations $x$. There are number of possible costfunctions that may be considered for this purpose. The fundamental principle of these is based on the statistical independence of the sources $s$. This paper uses FastICA algorithm for the sEMG source separation. FastICA algorithm is explained in brief next.

FastICA is a fixed point algorithm that employs higher order statistics for the recovery of independent sources [14, 15]. Separation is performed by minimization of negentropy of the mixture such that uncorrelated and independent sources whose amplitude distributions as non Gaussian as possible are obtained. FastICA can estimate ICs (independent components) one by one (deflation approach) or simultaneously (symmetric approach), and the extracted ICs can be lower than the number of mixtures so that the un-mixing matrix $\boldsymbol{W}$ can be rectangular. FastICA uses simple estimates of negentropy based on the maximum entropy principle [14].

\section{B. Suitability of ICA for decomposing sEMG}

The suitability of ICA to separate the signals originating from different sources is based on the following assumptions:

- The sources are independent.

- The sources are stationary (relative to the recorder),

- The signals are non-Gaussian, and

- Signal propagation delay is negligible.

Below are the arguments demonstrating that muscle electrical activity at low level of muscle contraction satisfies each of the above criteria.

- Each muscle is a set of motor units that is well separated from the other muscles and hence each muscle can be assumed to be an independent source.

- At low level of contraction, the muscle activity can be assumed to be made of independent motor unit action potentials (MUAP) that are individual pulses and thus the finite sum of these is non-Gaussian.

- At low level of muscle contraction, the length and position of the muscles with respect to the skin and other muscles remains stationary and hence with respect to the recording electrodes.
- Volume conduction in tissue is essentially instantaneous [16, 17].

Based on the above, the authors [18] have developed and reported the technique where the un-mixing matrix is estimated using ICA. Using this un-mixing matrix, sEMG recordings can be decomposed on an ongoing basis for a given electrode position. The vector consisting of RMS values averaged over the duration of the action are representative features of the action. Other researchers $[16,17]$ have reported success in the use of this approach to decompose EEG data.

\section{Methodology}

Experiments were conducted to evaluate the performance of the proposed system to identify finger and wrist flexion from sEMG recorded on the forearm. The RMIT University human experiments ethics committee approved the experimental protocol.

\section{Subjects}

Seven healthy subjects (six male and one female) volunteered to participate in this study. Mean age was 26.6 ( $\sigma$ $=2.05)$ years; mean weight $70.6(\sigma=6.56) \mathrm{kg}$; and mean height was $170.6(\sigma=7.42) \mathrm{cm}$. The participants inclusion criterion was; (i) no history of myo or neuro-pathology, and (ii) no evident abnormal motion restriction. Only right-hand dominant participants were included in this study. Each participant was given an oral and written summary of the experimental protocol and the purpose of the study and then was required to sign a consent form prior to participation.

\section{E. Experimental procedure}

Experiments were conducted where sEMG from the Flexor digitorum superficialis (FDS) muscle was recorded when the participant maintained specific finger flexion. FDS lies in the anterior compartment of the forearm, which has a primary function of flexing the digits in finger movements [19]. The two electrodes were placed on FDS muscle as shown in Fig.1. The force of contraction was measured using FlexForce sensor. The FlexiForce A201 (Tekscan, Boston, MA, USA) force sensor is an ultra-thin, flexible force sensor that can be fixed to measure the force of contraction from each of the fingers.

At the start of the experiment, the participant was made to generate maximum voluntary contraction (MVC) for 10 seconds and this was repeated 5 times. Based on the study of Basmajian and De Luca [1], the average of these five recordings was considered to be the MVC. Three different finger gestures were used as protocol to record sEMG from the participant: Middle finger flexion, Ring finger flexion and Little finger flexion.

The participant was asked to maintain each flexion for 7-8 secs for three different levels of forces i.e., $20 \%, 50 \%$ and $80 \%$ of MVC. The duration of each run of the experiment was 120 secs. The sampling rate for recording sEMG was 1024 samples/sec. The change in resistance of the FlexiForce is the measure of force of the sensor. To record the force exerted on the sensor, voltage across a fixed resistance in series with FlexiForce force sensor was recorded at 1024 samples/sec 
along with sEMG signal. Visual feedback of the force sensor output was given to the user to maintain steady muscle contraction.

\section{F. Data analysis}

Surface EMG is low frequency signal. EMG recording equipment (Delsys) filters the signal before the data acquisition. Low frequency noise components are eliminated during this process. The data was analyzed to compare the performance of ICA based sEMG separation and that of unseparated sEMG for identifying finger flexions for different MVCs. The length of each sEMG segment was approximately 2500 samples (2.5 seconds) and this corresponded to the duration of each action. To determine the efficacy of ICA, sEMG recordings were analyzed under the two conditions;

(i) when sEMG was not separated and,

(ii) when sEMG was separated using ICA

These are described below:

At the first step, RMS of the unseparated sEMG was computed for all the three different MVCs and each finger flextion. Next, sEMG was separated using fastICA and computing RMS for the separated muscle activity for each of the actions. Two channel sEMG data was separated using FastICA. Symmetric FastICA approach was used for sEMG source separation. Reason for this is that sEMG is a very low frequency signal and this method eliminates any unwanted artifacts in sEMG. The ICA separated data was used for RMS computation. However there exists an ambiguity problem in ICA. Hence a mitigation strategy to overcome shortcomings related to order and magnitude ambiguity related to ICA has been developed. This is achieved by using a combination of unmixing matrix obtained from FastICA corresponding to the specific user. This task was repeated for all the seven subjects who participated in the experiment. ICA and RMS were compared for their performance by determining the significance of separation of each flexions corresponding to different force levels of contraction. This analysis was repeated for all the three finger flexions and for all the seven subjects. The results were summarized in Table I.

The next step is to identify the separation patterns for 3 flexions during different force levels using Analysis of Variance (ANOVA) statistical anlaysis. ANOVA analysis was performed for all the three finger flexions and for all the participants. The mean result of ANOVA is explained in Table II.

Table I. Overall (mean) results for 20\%, 50\% and 80\% MVCs for all seven participants

\begin{tabular}{|c|c|c|c|c|c|c|c|}
\hline \multirow{4}{*}{ Ch1 } & & \multicolumn{2}{|c|}{$\mathbf{2 0 \%}$ MVC } & \multicolumn{2}{c|}{$\mathbf{5 0 \%}$ MVC } & \multicolumn{2}{c|}{$\mathbf{8 0 \%}$ MVC } \\
\cline { 2 - 8 } & Gestures & ICA RMS & RMS & ICA RMS & RMS & ICA RMS & RMS \\
\cline { 2 - 8 } & Little & 2.901 & 0.0953 & 5.462 & 0.0943 & 9.799 & 0.133 \\
\cline { 2 - 8 } & Ring & 3.167 & 0.0287 & 6.544 & 0.0347 & 10.125 & 0.151 \\
\cline { 2 - 8 } & Middle & 3.026 & 0.025 & 6.006 & 0.046 & 10.357 & 0.176 \\
\hline \multirow{3}{*}{ Ch2 } & Little & 3.054 & 0.0742 & 6.255 & 0.0821 & 10.092 & 0.139 \\
\cline { 2 - 8 } & Ring & 3.274 & 0.0279 & 6.501 & 0.0389 & 9.971 & 0.188 \\
\cline { 2 - 8 } & Middle & 3.234 & 0.0276 & 6.304 & 0.0458 & 10.361 & 0.172 \\
\hline
\end{tabular}

Table II. Overall (mean) p - Values using ANOVA for 20\%, $50 \%$ and $80 \%$ MVCs for all seven participants

\begin{tabular}{|c|c|c|}
\hline Gestures & ICA RMS & RMS \\
\hline Little & $3.8355 \mathrm{e}-11$ & 0.025 \\
\hline Ring & 0.00002 & 0.05 \\
\hline Middle & 0.00001 & 0.03 \\
\hline
\end{tabular}

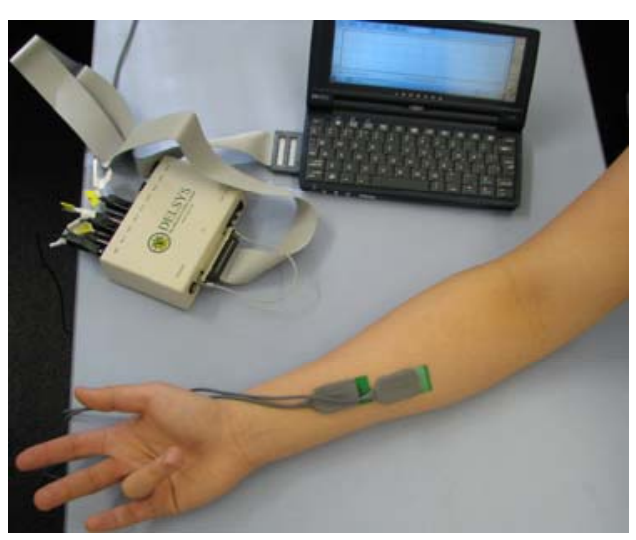

Fig.1 The experimental setup for finger flexion experiment

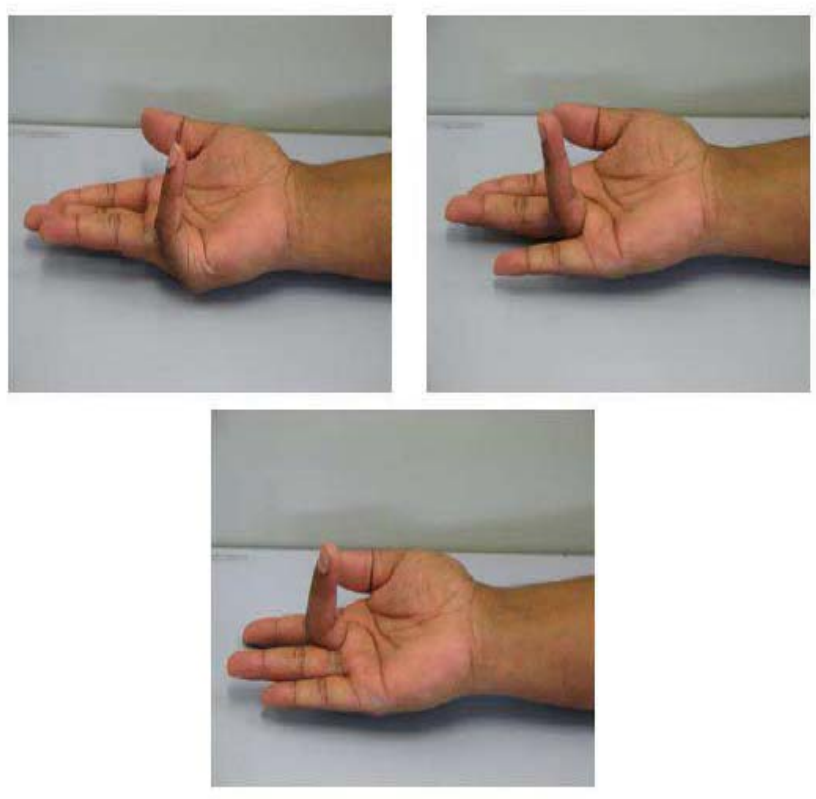

Fig.2 Three finger flexion performed during the experiment 


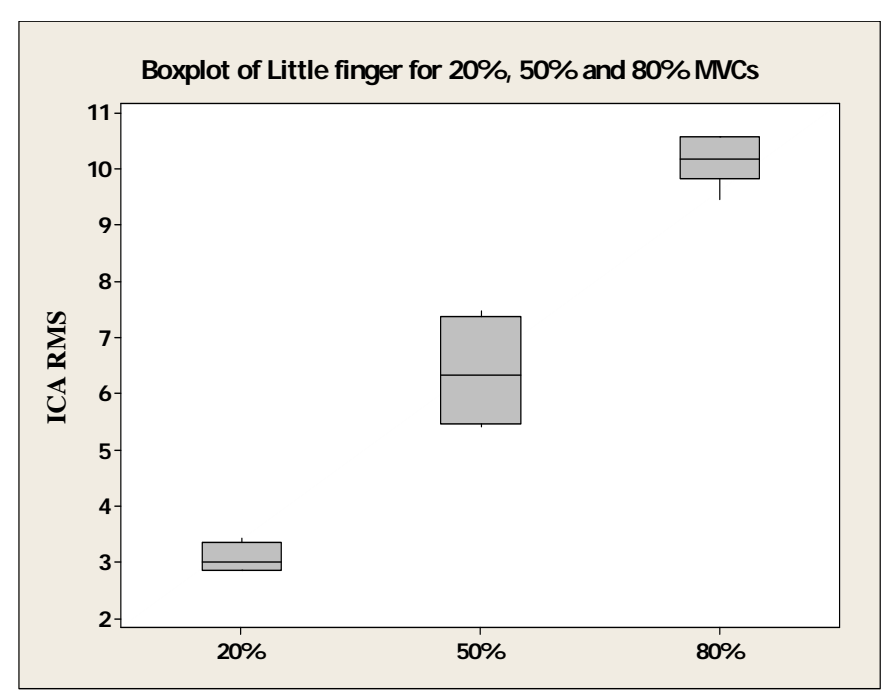

Fig.3 ICA-RMS Box plot of little finger for $20 \%, 50 \%$ and $80 \%$ MVCs

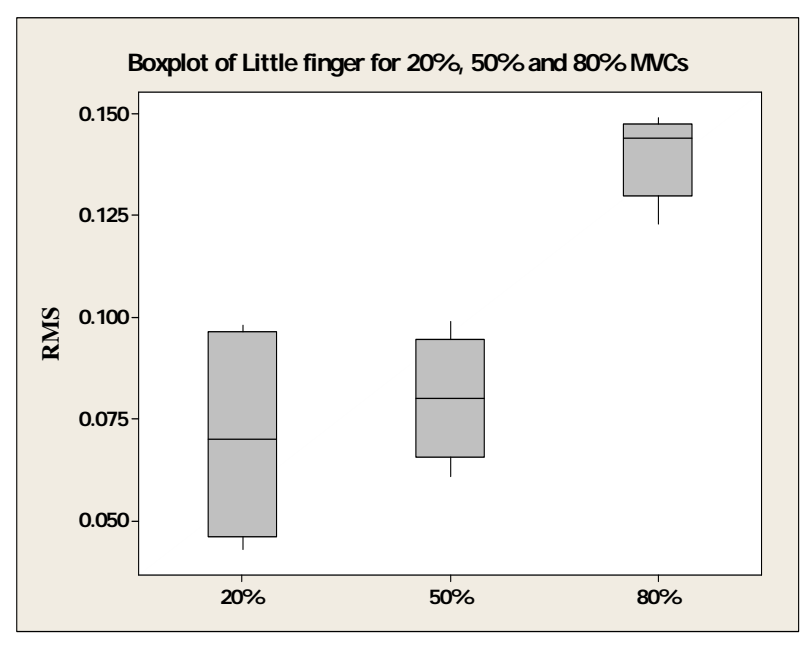

Fig.4 RMS Box plot of little finger for $20 \%, 50 \%$ and $80 \%$ MVCs

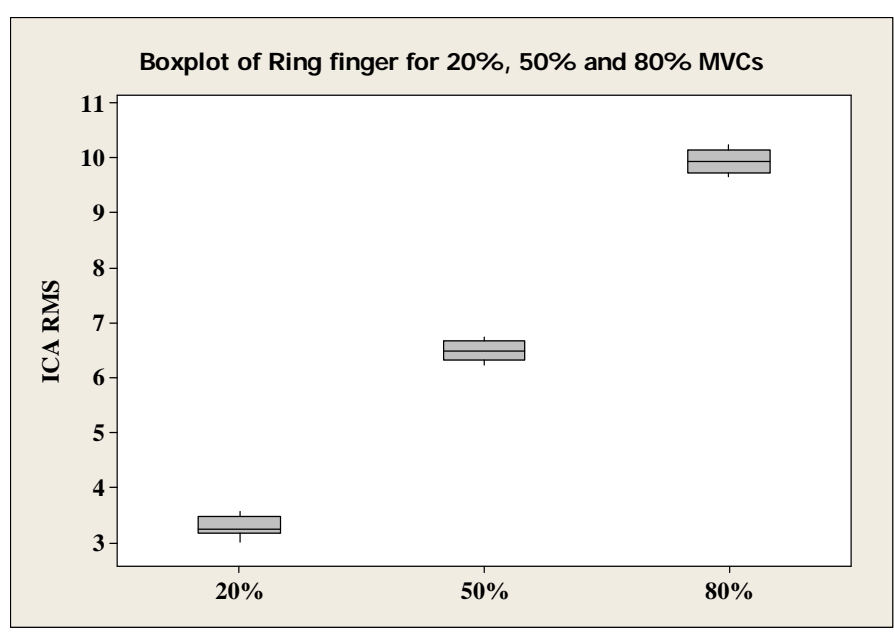

Fig.5 ICA RMS Box plot of middle finger for $20 \%, 50 \%$ and $80 \%$ MVCs

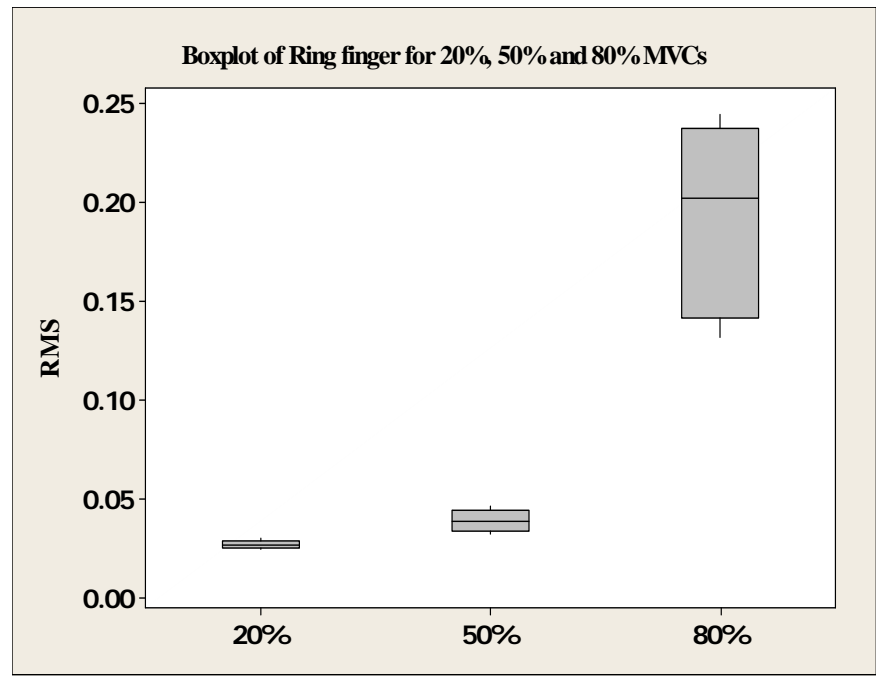

Fig.6 RMS Box plot of middle finger for $20 \%, 50 \%$ and $80 \%$ MVCs

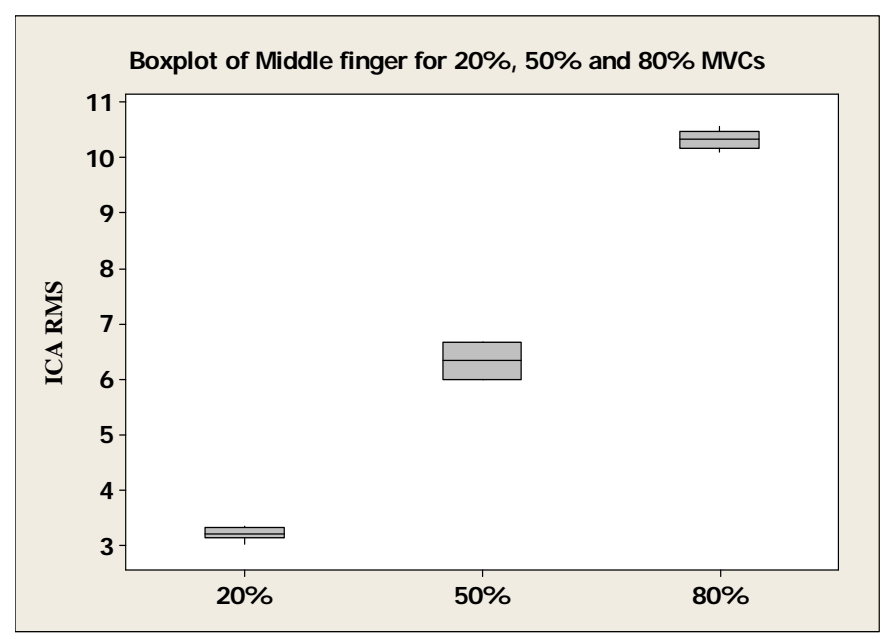

Fig.7 ICA RMS Box plot of ring finger for $20 \%, 50 \%$ and $80 \%$ MVCs

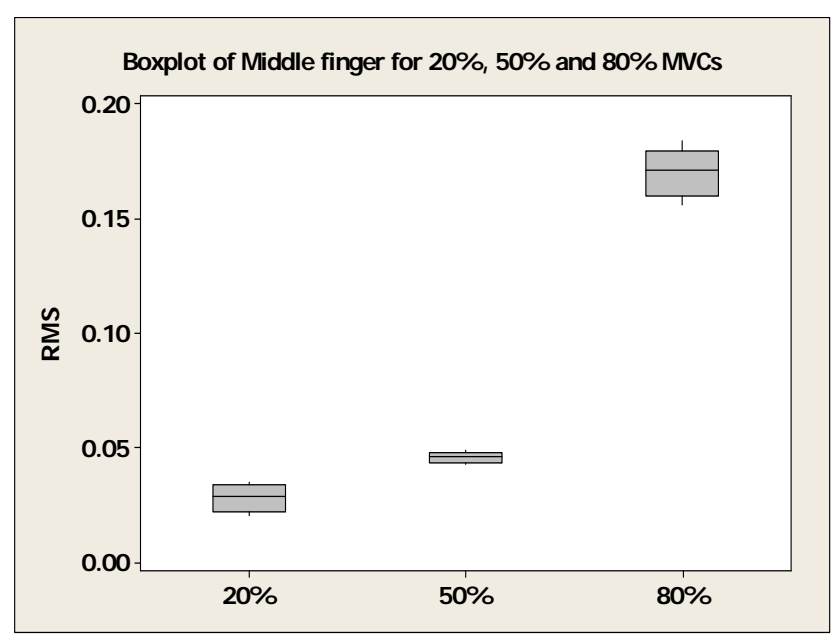

Fig.8 RMS Box plot of ring finger for $20 \%, 50 \%$ and $80 \%$ MVCs 


\section{RESULTS}

From Table I, it is observed that there is large increase in ICA RMS (mean values) for different finger flexion as the force of contraction increases from 20\% MVC to $80 \%$ MVC for all subjects. But the mean values of RMS have a marginal increase or no increase up to 50\% MVC.

The results show that values of RMS after ICA (ICA RMS) are a good indicator of the force of contraction of the muscles for all levels of muscle contraction. RMS of sEMG is an indicator of the force of contraction only when the level of contraction is high ( $80 \%$ MVC) but a poor indicator of the force of contraction when the level of contraction is low (20\%). This may be attributable to the effect of the background activity during low-level muscle activity.

In order to validate and visualize the data, the values were plotted using box plot to determine the difference in ICA RMS for flexions during different levels of contraction. Boxplots can be useful to display differences between populations without making any assumptions of the underlying statistical distribution [20]. The space between the different parts of the box helps indicate the degree of dispersion (spread) and skewness in the data, and identify outliers.

The boxplots for RMS and ICA separated sEMG for the flexion of the little finger at the three different levels of force of contraction for Subject 1 are shown in Fig.3 and Fig.4 respectively. From these plots, it is observed that ICA separated RMS is reliably able to differentiate between force of finger flexion - 20\%, 50\% and $80 \%$ MVC while this is not possible using RMS of sEMG.

Similarly, the plots for the Ring and Middle fingers flexion shown in Fig.5, Fig.6, Fig.7 and Fig.8, indicate that ICA RMS has more significant change than RMS, during low-level flexions with respect to the force levels of contraction.

\section{DisCUSSION AND CONCLUSIONS}

From the outcomes, it is evident that the patterns of sEMG against 3 different finger flexion actions for different MVCs were similar ( $>>0.01)$, while after separation using ICA, there is a clear identification of patterns $(p<0.0001)$ related to MVCs. This paper has compared the methods of identifying patterns at various levels of force (MVCs) using sEMG without separation and using sEMG after separation using ICA. The similar patterns for different MVCs using nonseparated sEMG are attributable to the high level of cross-talk, noise and low signal strength. ICA overcomes this shortcoming and the results clearly show that there is clear separation of patterns of sEMG during various levels of MVC

While this paper reports experiments conducted for three finger flexion action, this technique is suitable for the analysis of other actions and postures where cross-talk due to overlapping muscles is an issue such as when studying muscles of the lower back.

\section{REFERENCES}

[1] Basmajian, J.V., De Luca, C.J. (1985). Muscles Alive: Their Functions Revealed by Electromyography $\left(5^{\text {th }}\right.$ ed.). Baltimore, US: Williams \& Wilkins Publishers.
[2] Schlenzig, J., Hunter, E., Jain, R. (1994). Vision based hand gesture interpretation using recursive estimation. In Twenty-Eighth Asilomar Conference on Signals, Systems and Computers. IEEE, 1264-1271.

[3] Cheron, G., Draye, J.P., Bourgeios, M., Libert, G. (1996). A dynamic neural network identification of electromyography and arm trajectory relationship during complex movements. IEEE Trans. Biomed. Eng., 43, 552-558.

[4] Pavlovic, V.I., Sharma, R., Huang, T.S. (1997). Visual interpretation of hand gestures for human computer interaction. IEEE Trans. Pattern Anal. Mach. Intell., 19, 677-695.

[5] Wheeler, K.R., Jorgensen, C.C. (2003). Gestures as input: neuroelectric joysticks and keyboards. IEEE Pervasive Comput., 2 (2), 56-61.

[6] Koike, Y., Kawato, M. (1996). Human interface using surface electromyography signals. Electron. Comm. Jpn., 79 (9), 15-22.

[7] Doerschuk, P.C., Gustafson, D.E., Willsky, A.S. (1983). Upper extremity limb function discrimination using EMG signal analysis. IEEE Trans. Biomed. Eng., 30 (1), 18-28.

[8] Farry, K.A., Walker, I.D., Baraniuk, R.G. (1996). Myoelectric teleoperation of a complex robotic hand. IEEE Trans. Robot. Autom., 12 (5), 775-788.

[9] McKeown, M.J. (2000). Cortical activation related to arm-movement combinations. Muscle Nerve, 23 (9), S19-S25.

[10] McKeown, M.J., Radtke, R. (2001). Phasic and tonic coupling between EEG and EMG demonstrated with independent component analysis. J. Clin. Neurophysiol. , 18 (1), 45-57.

[11] Nakamura, H., Yoshida, M., Kotani, M., Akazawa, K., Moritani, T. (2004). The application of independent component analysis to the multichannel surface electromyographic signals for separation of motor unit action potential trains. J. Electromyogr. Kinesiol., 14 (4), 423-432.

[12] Peleg, D., Braiman, E., Yom-Tov, E., Inbar, G.F. (2002). Classification of finger activation for use in a robotic prosthesis arm. IEEE Trans. Neural Sys. Rehab. Eng., 10 (4), 290-293.

[13] Naik, G.R., Kumar, D.K., Singh, V.P., Palaniswami, M. (2006). SEMG for identifying hand gestures using ICA. In Workshop on Biosignal Processing and Classification at 2nd International Conference on Informatics in Control, Automation and Robotics, August 2006. Setubal, Portugal, 61-67.

[14] Hyvarinen, A., Karhunen, J., Oja, E. (2001). Independent Component Analysis ( $3^{\text {rd }}$ ed.). New York: John Wiley.

[15] Bell, A., Sejnowski, T. (1995). An information maximisation approach to blind separation and blind deconvolution. Neural Comput., 7, 1129-1159.

[16] Jung, T., Makeig, S., Lee, T., McKeon, M., Brown, G., Bell, A., Sejnowski, T. (2000). Independent component analysis of biomedical signals. In Second International Workshop on Independent Component Analysis and Blind Signal Separation, 633-644. 
[17] Makeig, S., Jung, T., Bell, A., Sejnowski, T. (1996). Independent component analysis of electroencephalographic data. Adv. Neural Inf. Proc., 8, 145-151.

[18] Naik, G.R., Kumar, D.K., Weghorn, H. (2007). Performance comparison of ICA algorithms for isometric hand gesture identification using surface EMG. In 3rd International Conference on Intelligent
Sensors, Sensor Networks and Information Processing, 3-6 December 2007, 613-618.

[19] Fridlund, A.J., Cacioppo, J.T. (1986). Guidelines for human electromyographic research. Psychophysiol., 23 (5), 567-589.

[20] Hair, J. F., Black, W. C., Babin, B.J., Anderson, R.E., Tatham, R. L. (2006). Multivariate Data Analysis. Prentice Hall. 